Anaesthesist 2012 $\cdot 61: 672-673$

DOI 10.1007/s00101-012-2069-2

Online publiziert: 10. August 2012

(c) Springer-Verlag 2012

\section{H. Metzler}

Univ.-Klinik für Anästhesiologie und Intensivmedizin, Medizinische Universität Graz

\section{Duale}

\section{Antiplättchentherapie und operative Medizin} Offene Fragen trotz Leitlinien?
Die interventionelle Kardiologie hat in den letzten Jahren einen der gewaltigsten Fortschritte der gesamten modernen Medizin gebracht. Durch die Entwicklung von Koronarstents konnten bei vielen $\mathrm{Pa}$ tienten mit koronarer Herzkrankheit eine signifikante Verbesserung ihrer Lebensqualität und eine Verlängerung eines relativ beschwerdefreien Lebens erreicht werden.

\section{$>$}

Die moderne interventionelle Kardiologie macht es uns nicht immer leichter.

Mit der Implantation von Koronarstents ist die Notwendigkeit einer Antiplättchentherapie, zumeist als duale Antiplättchentherapie, verknüpft. Gerade diese Fortschritte haben uns allerdings in der operativen Medizin mit neuen Herausforderungen und Problemen konfrontiert. Bei Patienten mit Koronarstents und dualer Antiplättchentherapie, die sich einem operativen Eingriff unterziehen müssen, bewegen wir uns oft auf einem schmalen Grat zwischen Blutung im Operationsgebiet und Stentthrombose im Koronargefäßsystem. Experten aus verschiedenen Fachdisziplinen, also v. a. Kardiologen, Anästhesisten, Operateure und Labormediziner, haben versucht, Leitlinien $\mathrm{zu}$ formulieren, die es uns im klinischen Alltag leichter machen, im konkreten Fall ein rationales Vorgehen zu begründen. Im Gegensatz zu kardiologischen Szenarien, wo evidenzbasier- te Empfehlungen auf der Basis großer Multizenterstudien mit nicht selten mehr als 10.000 Patienten existieren, führt das Vorliegen von zahlenmäßig kleineren Studien mit weniger Patienten, insbesondere bei nichtkardiochirurgischen $\mathrm{Pa}$ tientenkollektiven, zu weniger evidenzbasierten Strategien. Trotzdem ermöglichen die vorliegenden Empfehlungen für den klinischen Alltag i. Allg. ein sinnvolles Vorgehen. Allerdings werden dabei nicht Antworten auf alle klinischen Fragen gegeben werden können. In der vorliegenden Ausgabe berichten die Autoren Limper u. Lynch von einem Patienten mit liegendem Periduralkatheter, der ein akutes Koronarsyndrom entwickelt. Daraus ergaben sich grundsätzlich zwei mögliche Schritte: entweder der unverzügliche Beginn einer dualen Antiplättchentherapie mit Clopidogrel und Aspirin, verbunden mit der Notwendigkeit, irgendwann in den darauffolgenden Tagen den Epiduralkatheter zu entfernen, oder den Epiduralkatheter unverzüglich zu entfernen und erst danach mit der dualen Antiplättchentherapie zu beginnen. Die konkrete klinische Situation kam dem erstgewählten Vorgehen insofern entgegen, als sich bei Messung der Thrombozytenfunktion eine Clopidogrelresistenz ergab und damit ein risikoärmeres Entfernen des Periduralkatheters möglich machte. Hätte die Thrombozytenfunktionsmessung allerdings einen vollwirksamen Clopidogreleffekt ergeben, wäre das weitere Vorgehen wohl problematischer gewesen.
Die Autoren verweisen darauf, dass keine der existierenden Leitlinien die vorliegende Situation adressiert. Was nun die derzeit gültigen Empfehlungen der europäischen kardiologischen Gesellschaft betrifft, wird - etwas vereinfacht formuliert - bei akutem Koronarsyndrom eine sofortige Therapie mit in erster Linie Prasugrel bzw. Ticagrelor und in zweiter Linie Clopidogrel für zumindest 1 Monat und zusätzlich mit lebenslanger Aspirineinnahme empfohlen [1].

In den Empfehlungen der European Society of Anaesthesiology wird bezüglich Katheterentfernung bei neuroaxialer Blockade für Clopidogrel kein notwendiges Zeitintervall angegeben [2].

Kann man aus existierenden Leitlinien für eine konkrete Situation keine klaren Schlüsse ziehen, muss auf den wesentlichsten Punkt der gesamten Thematik „duale Antiplättchentherapie und perioperative Medizin“verwiesen werden.

\section{$>$}

Der Schlüssel zum richtigen Weg liegt im interdisziplinären Gespräch.

In allen unklaren und besonders bei schwierigen Situationen muss in gemeinsamer Absprache zwischen dem Kardiologen, dem Operateur und dem Anästhesisten entschieden werden. Im konkreten Fall hätten v. a. die interventionellen Kardiologen mit der Frage konfrontiert werden müssen, ob es nicht sinnvoll erschiene, eine antiischämische Therapie z. B. mit $\beta$-Rezeptoren-Blockern etc. zu star- 
ten, den Katheter zu entfernen und erst danach die duale Antiplättchentherapie zu beginnen. Die laufende Periduralanalgesie konnte offenbar das Auftreten der instabilen Angina pectoris nicht verhindern.

Naturgemäß präsentieren sich Kardiologen eher als beharrliche Anwälte ,ihres Koronarsystems“ und Operateure verständlicherweise eher als Anwälte „ihres Operationsgebiets“. Vielleicht mag sich gerade auch aus dieser Konstellation eine führende Rolle der Anästhesie als kompetenter Mittler zwischen beiden Standpunkten und als primärer Ansprechpartner für beide Seiten ergeben. Dies erscheint umso notwendiger, als die neuen P2Y12-Rezeptor-Hemmer Prasugrel und Ticagrelor, die stärker und schneller wirken - allerdings bei erhöhter Blutungstendenz - Clopidogrel teilweise verdrängen werden.

\section{Thrombozytenfunktions- messung - Ja oder Nein?}

Der Fallbericht wirft auch wieder die Frage des sinnvollen Einsatzes von neuen Thrombozytenfunktionsassays auf. Die Autoren des gerade in Der Anaesthesist publizierten Leitthemas fassen dazu recht prägnant die aktuelle Situation zusammen: Der Einsatz neuer Thrombozytenfunktionsassays kann vor der Manigfaltigkeit verfügbarer Testsysteme, der ungesicherten „cut offs“ und nicht zuletzt der negativen Ergebnisse der Studie Gauging Responsiveness with A VerifyNow ${ }^{\infty}$ Assay - Impact on Thrombosis And Safety (GRAVITAS) zur routinemäßigen Testung der Clopidogrelwirkung nicht empfohlen werden, bleibt aber ausgewählten Risikopatienten vorbehalten [3]. Dies ist auch in Übereinstimmung mit den demnächst publizierten „ESA Guidelines on the management of severe perioperative bleeding" [4].
Die Herausgeber der Zeitschrift Der Anaesthesist widmen jedenfalls diesem aktuellen Thema seit Jahren große Aufmerksamkeit und versuchen, durch Übersichtsarbeiten und Leitthemenbeiträge den Leser über den „state of the art“ kompetent und zeitaktuell zu informieren [3, $5,6]$.

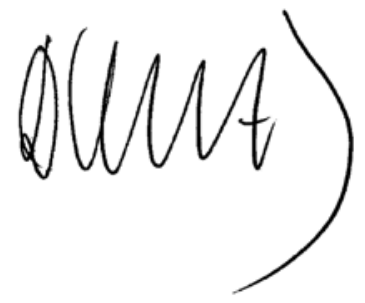

\section{H. Metzler}

\section{Korrespondenzadresse}

Prof. Dr. H. Metzler

Univ.-Klinik für Anästhesiologie und

Intensivmedizin, Medizinische Universität Graz

Auenbruggerplatz 29, $8036 \mathrm{Graz}$

Österreich

anaesthesie@meduni-graz.at

Interessenkonflikt. Der korrespondierende Autor gibt an, dass kein Interessenkonflikt besteht.

\section{Literatur}

1. Hamm CW, Bassand JP, Agewall S et al (2011) ESC Guidelines for the management of acute coronary syndromes in patients presenting without persistent ST-segment elevation. Eur Heart J 32:29993054

2. Gogarten W, Vandermeulen E, Van Aken H et al (2010) Regional anaesthesia and antithrombotic agents, recommendations of the European Society of Anaesthesiology, Eur J Anaesthesiol 27:9991015

3. Mahla E et al (2012) Thrombozytenfunktion - neue Medikamente, neue Assays. Anaesthesist 61:483496

4. Kozek-Langenecker SA et al (2012) Guidelines on the management of severe perioperative bleeding. ESA (in press)

5. Metzler $\mathrm{H}$ et al (2007) Koronarstents, duale Antiplättchentherapie und perioperative Probleme. Anaesthesist 56:401-412

6. Jambor C et al (2009) Perioperatives Management von Patienten mit Koronarstents. Anaesthesist 58:971-985

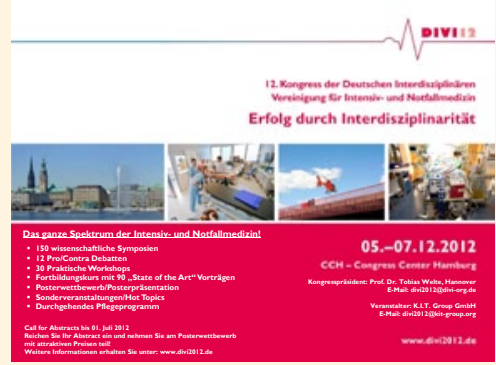

\section{DIVI 2012}

12. Kongress der Deutschen Interdisziplinären Vereinigung für Intensiv- und Notfallmedizin, 5.-7.12.2012 Congress Center Hamburg Kongresspräsident: Prof. Dr. Tobias Welte, Hannover

Erfolg durch Interdisziplinarität

- 150 wissenschaftliche Symposien

- 12 Pro/Contra Debatten

- 30 Praktische Workshops

- Fortbildungskurs mit 90 "State of the Art"-Vorträgen

- Posterwettbewerb/Posterpräsentation

- Sonderveranstaltungen/Hot Topics

- Durchgehendes Pflegeprogramm

E-Mail: divi2012@divi-org.de www.divi2012.de

Veranstalter: K.I.T. Group GmbH E-Mail: divi2012@kit-group.org 